\title{
LEDUC, Fernand, Vers les îles de lumière. Écrits (1942-1980). Montréal, Hurtubise-HMH, 1981. [9]-XXXVI-288 p. Présentation, établissement des textes, notes et commentaires par André Beaudet. 18,50 \$.
}

\section{Raymond Vézina}

Volume 36, numéro 4, mars 1983

URI : https://id.erudit.org/iderudit/304103ar

DOI : https://doi.org/10.7202/304103ar

Aller au sommaire du numéro

Éditeur(s)

Institut d'histoire de l'Amérique française

ISSN

0035-2357 (imprimé)

1492-1383 (numérique)

Découvrir la revue

Citer ce compte rendu

Vézina, R. (1983). Compte rendu de [LEDUC, Fernand, Vers les îles de lumière. Écrits (1942-1980). Montréal, Hurtubise-HMH, 1981. [9]-XXXVI-288 p.

Présentation, établissement des textes, notes et commentaires par André Beaudet. 18,50 \$.] Revue d'histoire de l'Amérique française, 36(4), 593-595. https://doi.org/10.7202/304103ar d'utilisation que vous pouvez consulter en ligne. 
LEDUC, Fernand. Vers les îles de lumière. Écrits (1942-1980). Montréal, Hurtubise-HMH, 1981. [9]-XXXVI-288 pages. Présentation, établissement des textes, notes et commentaires par André Beaudet. $18.50 \$$

Les écrits de Fernand Leduc font partie des quelque soixante-dix Cahiers du Québec publiés depuis 1971 sous la direction de Robert Lahaise, professeur d'histoire à l'UQAM. Tous les aspects de l'évolution du Québec, toutes les périodes de son histoire, toutes les ethnies ainsi que les personnalités exceptionnelles peuvent faire l'objet des Cahiers $d u$ Québec. Certains titres à succès comme La terre paternelle de Patrice Lacombe, inclus dans les programmes des CEGEP, ont connu jusqu'à quatre éditions. Une quarantaine de manuscrits attendent actuellement une amélioration de la situation financière pour faire leur apparition sur les rayons des librairies.

La collection de Textes et documents littéraires - l'une des 16 collections des Cahiers du Québec - s'est enrichie d'un titre important grâce au travail d'André Beaudet. Écrivain et professeur à l'UQAM, André Beaudet s'est lié d'amitié avec Fernand Leduc au cours d'un séjour d'études à Paris de 1976 à 1978. Quelques articles dans les journaux et un certain nombre d'expositions individuelles n'ayant pu faire connaître au public canadien cet artiste québécois installé en France depuis 1947, l'entreprise d'André Beaudet reçut l'appui ferme du directeur de la collection. 
À peine une poignée de spécialistes ayant lu les articles de Bernard Teyssèdre s'intéressaient alors à l'évolution de Fernand Leduc. Publié en 1969, un premier article analysait les débuts de Leduc (automne 1941-février 1947) en regard du surréalisme et de Borduas ${ }^{1}$. En 1971, Teyssèdre signait un autre texte important consacré aux automatistes de Montréal dans le catalogue d'une exposition du Musée d'art contemporain $^{2}$. Face à un académisme stérile, le mouvement automatiste était présenté comme une expérience de type romantique où le lyrisme, la spontanéité, les hasards et même l'indiscipline deviennent sources de nouveautés. A côté de Fernand Leduc appelé le «théoricien du groupe», l'auteur alignait les noms suivants: Paul-Émile Borduas, Pierre Gauvreau, JeanPaul Mousseau, Jean-Paul Riopelle, Marcel Barbeau et Marcelle Ferron.

L'étude de Jean-Pierre Duquette publiée en 1980, fit ensuite apparaître la véritable importance de Fernand Leduc, alors âgée de 64 ans, en fournissant une vue d'ensemble de sa carrière ${ }^{3}$. L'auteur s'attarde à chaque étape de l'évolution de l'artiste, fournit la liste de 18 expositions individuelles présentées de 1950 à 1980 et fait état des expositions de groupe. En 1980 également, le Musée d'art contemporain de Montréal présentait une importante exposition centrée sur les microchromies de Leduc.

Troisième étape dans les études Fernand Leduc, le livre d'André Beaudet sera utile d'abord à tous ceux qui veulent des documents de première main.

Le volume comprend trois parties: une présentation couvrant les pages I à XXXVI; les textes de Leduc des pages 1 à 221; une suite de 392 notes couvrant les pages 223 à 288 . Il est maintenant possible d'apprendre de Fernand Leduc lui-même quels sont les véritables motifs de ses nombreux «refus». Dès 1943, refus de vivre au sein d'une société qui marque le pas au point de considérer comme anormaux les intellectuels actifs ${ }^{4}$. Mars 1947, refus d'accepter les orientations surréalistes d'André Breton pour qui les intentions de l'artiste étaient prépondérantes ${ }^{5}$. Leduc, ayant affirmé sa foi en «l'authenticité et la gratuité des oeuvres sans considération pour leur résultat» ${ }^{6}$, allait lui-même être rejeté par Breton à l'issue de leur entretien à Paris. Août 1948, Refus global, qui pour Leduc, marque le rejet de l'automatisme ${ }^{7}$. En 1055, refus de suivre les voies définies par Borduas ${ }^{8}$. Depuis 1970, refus des formes, des con-

\footnotetext{
1 Bernard Teyssèdre, «Fernand Leduc, peintre et théoricien du surréalisme à Montréal», dans La Barre du jour (Montréal, janvier-août 1969): 224-270.

2 Bernard Teyssèdre, «Les automatistes de Montréal», dans Borduas et les Automatistes. Montréal 1942-1955 (Montréal, Musée d'art contemporain, 1971): 9-15.

3 Jean-Pierre Duquette, Fernand Leduc (La Salle, Hurtubise-HMH, 1980), 154 pages.

4 Fernand Leduc, «L'artiste, un être anormal?», dans Le Quartier Latin (3 décembre 1943): 5.-Beaudet, 10-12.

5 Lettre de F. Leduc à Monsieur et Madame Paul-Émile Borduas, Paris, 22 mars 1947. - Beaudet, 46-49.

6 Beaudet, Fernand Leduc, 233, note 67. Ibid., 200. 153-155.

8 Fernand Leduc, «Leduc vs Borduas», dans l'Autorité, 4 mars 1955. - Beaudet,
} 
trastes de couleurs et de valeurs afin de se concentrer tout entier sur le rendu de la lumière ${ }^{9}$.

Le travail d'André Beaudet servira également ceux qui s'intéressent aux multiples composantes de l'automatisme. Nous possédons maintenant la possibilité de pousser plus loin l'analyse des courants internationaux qui ont agi sur les automatistes même après la disparition du groupe. Alors que Louise Renaud et plus tard Borduas avaient parlé des surréalistes européens installés à New York, Leduc a analysé, décrit et commenté tout ce qui se passait à Paris pour le bénéfice de ses amis peintres à Montréal. Même les textes récents ${ }^{10}$ évoquent l'automatisme et proposent des explications en tenant compte de personnalités comme André Breton, Chirico, Dali, Arp, Matta ou Miro. En fait, Beaudet estime que près des deux tiers des textes de Leduc parlent de l'automatisme.

Il aurait fallu mettre davantage en valeur les textes inédits et faire ressortir la rareté de certains textes publiés. En fait, il est même difficile de savoir quels sont les textes inédits soit que l'on consulte le Sommaire où le mot «inédit» apparaît à la page (sans pagination) qui précède la Présentation, soit que l'on consulte la Bibliographie où le même mot apparaît à la page XXXVI. Le lecteur aimerait par ailleurs trouver à la fin de chaque texte antérieurement publié la référence bibliographique sans devoir consulter constamment les notes. Les entretiens avec Leduc et Thérèse Renaud se détachent mal du texte d'André Beaudet lui-même. Le nom de Thérèse Renaud mis en évidence à la page XXVIII laisse même croire que la présentation entière est issue de sa plume d'autant plus que le nom de l'auteur n'apparaît pas à la page XXXVI.

Après les textes suggestifs de Bernard Teyssèdre et de Jean-Pierre Duquette, le recueil d'André Beaudet fournit un ensemble de sources précieuses non seulement pour l'étude de Fernand Leduc mais pour la compréhension de l'ensemble du mouvement automatiste lui-même étape charnière de l'évolution culturelle québécoise au moment où s'amorçait déjà la Révolution tranquille. 